\title{
Substructure Coupling with Joint Identification for Reconfigurable Manufacturing Systems
}

\author{
J. Chae, S. S. Park*, S. Lin \\ Department of Mechanical and Manufacturing Engineering \\ University of Calgary, Calgary, Alberta, Canada T2N 1N4 \\ *sipark@ucalgary.ca
}

\begin{abstract}
Today's manufacturing industries require rapid production of small batch sized products with great accuracy and productivity. When compared with traditional dedicated systems, reconfigurable manufacturing systems (RMS) offer rapid change in system configurations, machines and controls to adjust to flexible demands. This can be achieved by combining modular substructures together with different functionalities depending on requirements. In this study, a method of assembling the known dynamics of substructures is investigated through the receptance coupling approach. The classical receptance technique is enhanced by identifying the joint dynamics between substructures through experimental and finite element (FE) analyses. This identification method also includes the translational and rotational degrees of freedom responses, which represent the mass, spring, and damping elements of the joint. The determination of rotational responses can be very challenging, and the proposed method solves the rotational responses with two separate experimental measurements using gauge tools. This novel identification method overcomes the limitations posed by other identification methods, by minimizing numerical errors and problems associated with convergence. Experimental tests, using a fastener joint, were performed to verify the effectiveness of the joint identification method.
\end{abstract}

\section{Introduction}

Today's manufacturing industries are faced with many challenges in order to deliver high quality, flexible, cost-effective products. Products are becoming more complex to accommodate demand, and their life cycle periods are getting shorter, more than ever before. To preserve competitive performance in manufacturing industries, the infrastructure of production plants should be able to adapt in order to respond to diverse market demands. The established Dedicated Manufacturing Systems (DMS) have advantages in fabricating a specific part at a mass production volume over a long period of time. When demand exceeds supply and operating at full capacity, DMS are cost effective. Conversely, Flexible Manufacturing Systems (FMS) are economical when the production volumes are low and a large variety of parts are produced [1]. However, situations in which DMS do not operate at full capacity and the lack of robustness in FMS with redundant resources are the vital drawbacks to the traditional manufacturing systems in modern enterprises. Consequently, a new scheme for manufacturing - Reconfigurable Manufacturing Systems (RMS) - is being introduced to meet the challenges and deliver high quality, flexible, and cost-effective products that fulfill production and operation requirements.

For the economical accommodation of diverse demands, RMS combines the merits of both DMS and FMS by adapting an open-architecture control. In software, an open architecture allows for reconfiguration of the controller; and in machine hardware, modular machine tools offer flexible machining options [2]. Software control can be modified to interface with the specific machine tools selected and perform the distinct assignment. Therefore, RMS exhibits the key characteristics of modularity, convertibility, customization, integrability and diagnostics [3], and accommodates expeditious market demands effectively with adjustable capacity and functionality. RMS can, thus, be reconfigured around a part family and can offer customized flexibility at lower cost without any waste of resources. RMS is the key to future manufacturing, but advances 
in manufacturing will not occur without the proper machine tools and equipment. Hence, there is a demand for the fundamental change of machine tools in terms of their structure (modular structure), components (controllers, hardware/software, spindles, tooling), and sensors. Consequently, the development of new theories, design concepts, and methodologies is needed [3]. These innovations are essential to the assembly of the forthcoming Reconfigurable Manufacturing Tools (RMT).

\subsection{Roles of joints in RMS}

RMS consists of a variety of replaceable modules, which depend on the operation process. The interfaces between the modules are leading factors of RMS. The objectives of mechanical interfaces are the transmission of forces and moments, and alignment between elements and modular structure. Dynamic connections allow for the attachment and detachment of various tools, while fixed interfaces are used for connections only to be disengaged in case of system configuration. RMT empowers the modularity of being able to simply remove, add or change the constituent units or modules of the system or the machine [2]. The modular components in the RMS can be arranged and rearranged with joints (i.e. fastener and pin/hole). Such advanced enabling technology requires the establishment of exquisite mechanical interfaces that join exchangeable modules while maintaining the dynamic stiffness of the machine tool. The design of joining methods is aimed at enhancing the following performance criteria: repeatability, precision/reliability, stiffness and rampup time to minimize the cost and maximize the proficiency of RMT. A number of joints such as Tslot, adjustable stops, V-block, clamps and springs can be mounted to the main structure and provide a fixture [4]. Followed by joint development, various methodologies have been established to investigate the performance of the joining.

One of the key criteria of RMS is to achieve high stiffness and damping of machine tool structures, so that accurate parts can be fabricated. Therefore, the prediction of modular structure dynamics is important, especially for machining operations where identification of chatter-free cutting conditions is paramount to productivity. In general, a system consists of different substructures with distinctive joints; and, the response of the global system is based on the dynamic characteristics of each subsystem. The connections, such as welds, bolts, rivets and bearings, between coupled subsystems also play a significant role in the response of the global system, due to different material properties, geometry, and contact area and methods. Dynamic stiffness and damping are two critical elements to the identification of the characteristics of a system. High stiffness and damping suggest precision in the fabricated parts in RMS. The dynamic stiffness is proportional to the natural frequency, which is the critical property related to the frequency range of the work force. When the natural frequency lies within the frequency range of the work force, damage to the joints and the system occur due to resonance. Hence, the frequency range of the work force for a system is limited by the natural frequency of the specific system. The existence of joints in a system also yields damping and dissipation of energy. Thus, for a RMS consisting of a number of various modular components for a broad range of operations, the damping is cumulative and increases due to the number of joints in RMS.

Joints in the system cannot be tested easily due to limited access. A number of approaches apply an analytical or numerical analysis method (i.e. finite element method) to establish the dynamic analysis of a RMS. Finite element (FE) analysis provides reliable results for singular modular structures; however, the dynamics of the overall RMS cannot be constituted as a composition of each individual module [5]. Moreover, most of the analyses were performed based on the assumption of rigid structure, neglecting the effect of joints on the system. This can cause severe deviations in dynamics between theoretical predictions and the actual systems, possibly with detrimental results. Even if the joints can be separated, changes in boundary conditions alter the dynamics of joint parameters; therefore, the identified parameters of a joint in certain test environments cannot apply to other environments.

A number of researchers have tried and presented several methods of identification for the dynamic characteristics of many kinds of joints from various perspectives. Wang and Sas [6] constructed a simple free-free system, consisting of two mass blocks joined by an elastic element, and identified the physical characteristics (i.e. stiffness and damping) of joints experimentally, including the Rotational Degree of Freedom (RDOF). Shen and Astaneh-Asl [7] modeled the bolt connection as a semi-rigid joint, and performed cyclic tests on the connections and observed its hysteresis behavior. From the tensile test, the stiffness of the joints was obtained from the deformation and force graph, which displays the hysteresis loop. The result suggested that the value of the stiffness is related to the incremental force. Ma et al. [8] modeled the bolted joint as a spring element and evaluated its dynamics based on the comparison of the overall dynamics of the bolted system to the unbolted system. The majority of the joint identification 
methods relied on numerical simulations or static analysis in order to obtain the stiffness constant of the joints. They often ignored the damping and mass effects of the joints and focused on the effect of the joints in the global system. Ignoring the mass of the joints can lead to incorrect evaluations of their dynamics because they consist of several parts such as pins, bolts, adhesive materials, etc.; therefore, the mass must be considered in the joint identification.

The objective of this paper is to develop a joint identification method using receptance coupling to obtain and evaluate the dynamic responses of various joints, especially in RMS, with experimental and FE data. The technique enables designers to predict overall modular machine dynamics, which consist of spindle, drive systems, workpiece and tools, prior to the actual building of the reconfigurable machine. It also allows designers to optimize the design to enhance the dynamic performances of RMS. Furthermore, this method can effectively increase the performance of machining productivity and accuracy by minimizing chatter vibrations in machining processes.

The paper is organized in the following manner. First, this paper presents a novel methodology for predicting the Frequency Response Functions (FRF) of the assembled system with some necessary assumptions based on the receptance coupling techniques. Second, the difficulty of obtaining FRF in RDOF is overcome through the combination of analytical and experimental methods. Third, the dynamic characteristics of a joint in both the Translational Degree Of Freedom (TDOF) and RDOF are obtained by the enhanced receptance coupling techniques. Fourth, the paper demonstrates its application with experiments identifying the dynamic characteristics of the joint. The paper concludes with the possible applications and contributions of this approach.

\section{Methodology}

The Frequency Response Functions (FRF) of the machine structure play important roles in the prediction of system behavior and instabilities, such as regenerative chatter [9]. The essence of the proposed approach is to verify the effects of joints by the comparison of FRF between the dynamics of the rigid structure and the joined structure with the identical dimensions. In this study, a classical receptance coupling technique is enhanced by proposing a method to identify joint dynamics. To identify the coupling, the receptance coupling method is used at every increment of frequency with the experimental measurements and FE data.

Figure 1 depicts the algorithm of the proposed joint identification approach. The identification method consists of three steps. The first step is to analytically identify the rotational dynamics at Substructure A either through FE analysis or beam formulas. It is rather difficult to experimentally measure the dynamics of angular displacements by applying a moment and force at Substructure B. The rotational dynamic response of Substructure B is determined by substituting the direct and cross FRF measurements taken at the free end of the assembly and the rigid cylinder in the receptance coupling expressions, as explained in the authors' previous work [10]. The second step is to acquire joint dynamics of the fastener joint by comparing the FRF of the rigid system and the joined system. The joined system consists of two substructures: a spindle and a short rigid tool (Substructure B) whose dynamics are experimentally obtained through experimental modal analysis (EMA); and, an arbitrary tool (Substructure A) whose dynamics are analytically obtained through FE (finite element) analysis.

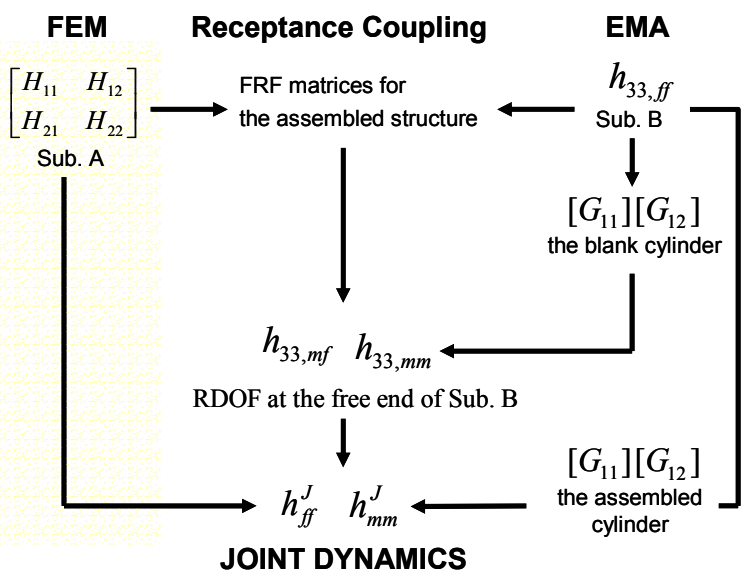

Figure 1. Algorithm of the joint identification method

\subsection{Receptance coupling}

Receptance is the relationship between the displacement $x(\omega)$ of the system and the force $F(\omega)$ in frequency domain based on the assumption that the response of the system is linearly elastic. The receptance coupling method enables two substructures to be joined as shown in Figure 2, where $f_{1}, f_{2}$ and $f_{3}$ are applied forces; $x_{1}, x_{2}$ and $x_{3}$ are displacements; $f_{2}^{J}, f_{3}^{J}$, $M_{2}^{J}$ and $M_{3}^{J}$ are forces and moments at joint; and, 
$k_{x}, k_{\theta}, c_{x}$ and $c_{\theta}$ are stiffness and damping constants in TDOF and RDOF [11][12]. The receptance coupling method is employed to correlate experimental and analytical data rather than the component mode synthesis (CMS) approach, because CMS requires accurate mode shapes that are often difficult to obtain from experimental measurements. One of the concerns in this experimental analysis is that the measured data may be contaminated with surrounding noises, especially during assessment of the impact forces.

Substructures A and B can represent any substructures that are connected by the joint. Considering the particular test environment in this paper, the system can represent a RMT; therefore, Substructure A can represent drill, end mill, etc., and Substructure B can represent the tool holder or spindle of the computer numerically controlled (CNC) machine. The relationship of the displacement and the force is characterized by the system FRF. The responses of the unassembled Substructures A and B are given by:

$$
\begin{aligned}
& {\left[\begin{array}{l}
x_{1} \\
x_{2}
\end{array}\right]=\left[\begin{array}{ll}
H_{11} & H_{12} \\
H_{21} & H_{22}
\end{array}\right]\left[\begin{array}{c}
f_{1} \\
f_{2}+f_{2}^{J}
\end{array}\right]} \\
& {\left[x_{3}\right]=\left[H_{33}\right]\left[f_{3}+f_{3}^{J}\right]}
\end{aligned}
$$

where, $H_{i j}$ is the FRF of the substructures. Subscripts $i$ and $j$ indicate the location of the displacement and force, respectively.

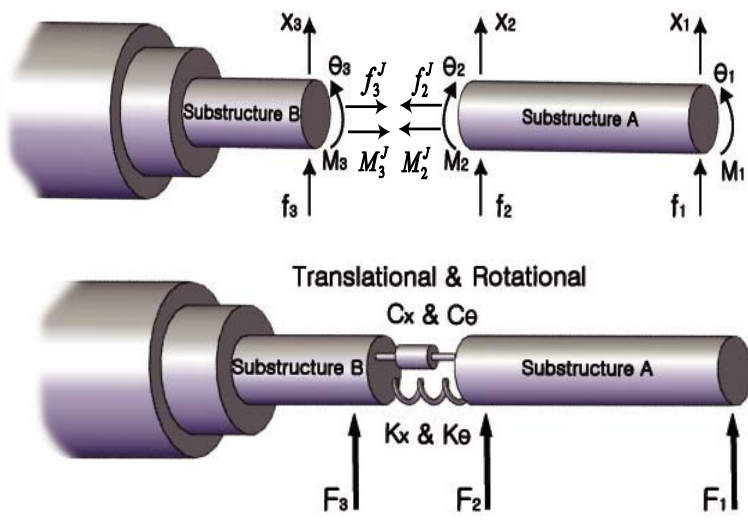

Figure 2. Receptance coupling of two substructures

Two substructures are assembled together by a joint, which is expressed by stiffness, damping, and mass elements. The assembled system is displayed in
Figure 2. The equilibrium and compatible conditions at the joint must be satisfied in the assembled structure, as shown in Equations 2 and 3, respectively.

$$
\begin{aligned}
& f_{2}^{J}+f_{3}^{J}=0, M_{2}^{J}+M_{3}^{J}=0 \\
& m\left(\ddot{x}_{3}-\ddot{x}_{2}\right)+c\left(\dot{x}_{3}-\dot{x}_{2}\right)+k\left(x_{3}-x_{2}\right)=f_{2}^{J} \\
& J\left(\ddot{\theta}_{3}-\ddot{\theta}_{2}\right)+c\left(\dot{\theta}_{3}-\dot{\theta}_{2}\right)+k\left(\theta_{3}-\theta_{2}\right)=M_{2}^{J}
\end{aligned}
$$

where, $m$ or $J, k$ and $c$ represent the mass, stiffness, and damping matrices of the joint, respectively. By applying the Laplace transform to Equation 3, we can obtain Equation 4 as:

$$
\begin{aligned}
& {\left[\begin{array}{l}
\left(x_{3}-x_{2}\right) \\
\left(\theta_{3}-\theta_{2}\right)
\end{array}\right]=\left[\begin{array}{cc}
\frac{1 / m}{s^{2}+2 \varsigma_{f f f} \omega_{h, f f} s+\omega_{n, f f}^{2}} & 0 \\
0 & \frac{1 / J}{s^{2}+2 \varsigma_{m m} \omega_{n, m n} s+\omega_{h, m m}^{2}}
\end{array}\right]\left[\begin{array}{l}
f_{2}^{J} \\
M_{2}^{J}
\end{array}\right]} \\
& =\left[\begin{array}{cc}
h_{f f}^{J} & 0 \\
0 & h_{m m}^{J}
\end{array}\right]\left[\begin{array}{l}
f_{2}^{J} \\
M_{2}^{J}
\end{array}\right]=\left[H^{\prime}\right]\left[\begin{array}{l}
f_{2}^{J} \\
M_{2}^{J}
\end{array}\right]
\end{aligned}
$$

where, $H^{J}$ represents the translational and rotational joint dynamics. In this paper, cross-coupled properties (i.e. $h_{f m}^{J}$ and $h_{m f}^{J}$ ) of the joint between TDOF and RDOF dynamics are assumed to be zero. It has already been verified by Wang et al. [6] that the crosscoupled terms of joints (i.e. $h_{f m}^{J}$ and $h_{m f}^{J}$ ) have no effect on the response of the assembled system. The performance of the proposed algorithm can be effectively improved by excluding the cross-coupled properties of the joint and reducing the computational time on the analysis of the dynamics of the machine tool system.

Substituting Equations 1 and 2 into Equation 4 to obtain Equation 5:

$$
\begin{aligned}
f_{2}=-\left(H_{22}+H_{33}+H^{J}\right)^{-1} H_{2} f_{1}-( & \left(H_{22}+H_{33}+H^{J}\right)^{-1} H_{22} f_{2} \\
& +\left(H_{22}+H_{33}+H^{J}\right)^{-1} H_{33} f_{3}
\end{aligned}
$$

The matrix $\left(H_{22}+H_{33}+H^{J}\right)$ must be invertible, which suggests the determinant of the matrix is not zero. By substituting Equation 5 into Equations 1, the FRF matrices for the assembled structure are constructed:

$\left[\begin{array}{l}X_{1} \\ X_{2} \\ X_{3}\end{array}\right]=\left[\begin{array}{ccc}G_{11} & G_{2} & G_{3} \\ G_{21} & G_{22} & G_{33} \\ G_{31} & G_{22} & G_{33}\end{array}\right]\left[\begin{array}{l}F_{1} \\ F_{2} \\ F_{3}\end{array}\right]$

$\left[H_{11}-H_{12}\left(H_{22}+H_{33}+H^{\prime}\right)^{-1} H_{21} H_{12}-H_{12}\left(H_{22}+H_{33}+H^{\prime}\right)^{-1} H_{22} \quad H_{12}\left(H_{22}+H_{33}+H^{\prime}\right)^{-1} H_{33}\right]\left[F_{1}\right.$ $=H_{21}-H_{22}\left(H_{22}+H_{33}+H^{\prime}\right)^{-1} H_{21} H_{22}-H_{22}\left(H_{22}+H_{33}+H^{\prime}\right)^{-1} H_{22} \quad H_{22}\left(H_{22}+H_{33}+H^{\prime}\right)^{-1} H_{33} \quad F_{2}$ $\left.H_{33}\left(H_{22}+H_{33}+H^{\prime}\right)^{-1} H_{21} \quad H_{33}\left(H_{22}+H_{33}+H^{\prime}\right)^{-1} H_{22} \quad H_{33}-H_{33}\left(H_{22}+H_{33}+H^{\prime}\right)^{-1} H_{33}\right]_{3}\left[\begin{array}{l}F_{3} \\ .\end{array}\right.$ 
where, $G_{i j}$ represents the FRF of the assembled system.

From Equation 6, the FRF matrix of any points in Figure 2 can be determined. As a result, the FRF matrices of each substructure are required to construct the matrix of Equation 6. Fortunately, the FRF matrix can be evaluated by experimental modal analysis (EMA) or the FE (finite element) method. The RDOF is a critical element in the receptance coupling [10]; therefore, the FRF matrix of the each substructure is represented by translational displacement $(x)$ as well as rotational displacements $(\theta)$ with force $(f)$ and moment $(M)$, as shown in Figure 2.

$\left[\begin{array}{c}x_{1} \\ \theta_{1}\end{array}\right]=\left[\begin{array}{ll}h_{11, f f} & h_{11, f m} \\ h_{11, m f} & h_{11, m m}\end{array}\right]\left[\begin{array}{c}f_{1} \\ M_{1}\end{array}\right] \Rightarrow\left\{x_{1}\right\}=\left[G_{11}\right]\left\{F_{1}\right\}$

By substituting Equation 7 into Equation 6, the FRF of Location 1, which includes the translational and rotational degrees-of-freedom, is obtained as:

$$
\begin{aligned}
& {\left[\begin{array}{ll}
G_{11, f f} & G_{11, f m} \\
G_{11, n f} & G_{11, n m}
\end{array}\right]=}
\end{aligned}
$$

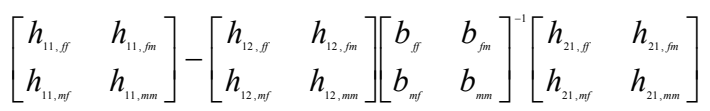

where,

$$
\begin{aligned}
& {\left[\begin{array}{ll}
b_{g f} & b_{f m} \\
b_{m f} & b_{m n}
\end{array}\right]^{-1}=}
\end{aligned}
$$

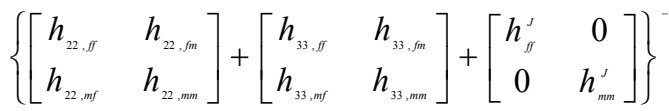

where, $h_{f f}^{J}$ (translation) and $h_{m m}^{J}$ (rotation) describe the dynamic characteristics of the joint.

The assembled dynamics are formulated with the receptance matrix of each substructure (A, B), based on the equilibrium and compatibility conditions. Furthermore, the assembled dynamics also includes the joint dynamics.

\subsection{Finding the RDOF responses at Substructure B}

To determine RDOF responses experimentally is very difficult [13][14] due to the inability to accurately measure rotational displacements. Often, designers ignore the rotational dynamics during the substructure coupling processes [10]. However, this negation of RDOF responses leads to severe inaccuracy in the coupled dynamics. To overcome the difficulties associated with measuring the RDOF responses, the authors have proposed indirect methods to obtain the rotational dynamics at Location 3 in Figure 2, based on gauge blank cylinders as described in the reference
[10]. This identification first considers the joint between the substructure as rigid by assuming the $h_{f f}^{J}$ (translation) and $h_{m m}^{J}$ (rotation) terms equal to zero and, then, indirectly identifies the rotational dynamics at the free end of Substructure B $\left(h_{33, \text { fm }}=h_{33, m f}, h_{33, m m}\right)$, as illustrated in the reference [10].

\subsection{Finding joint dynamics}

In Equation 9, all the FRF matrix elements in RDOF and TDOF for Substructures A and B are obtained except the joint dynamics, which can be determined from EMA and the FE method. The elements in the first row and first column of matrices $\left[G_{11}\right]$ and $\left[G_{12}\right]$ can be measured directly from the EMA of the assembled system. Therefore, two equations and two unknown parameters $\left(b_{f f}\right.$ and $\left.b_{m m}\right)$ are presented in Equations 10 and 11. Using the symbolic non-linear analytical toolbox [15], $b_{f f}$ and $b_{m m}$ can be identified based on two equations:

$u=a+\frac{f(-\beta \sigma+e k)+c(-\beta e+\delta \sigma)}{\beta^{2}-\delta k}$
$v=\sigma+\frac{g(-\beta \sigma+e k)+d(-\beta e+\delta \sigma)}{\beta^{2}-\delta k}$

where,

$G_{11, f f}=u, G_{12, f}=v, h_{11, f}=a, h_{12, f f}=\sigma$,

$h_{21, f f}=c, h_{22, f f}=d, h_{12, n f f}=e, h_{21, n f}=f$,

$h_{22, n f}=g, b_{f f}=k, b_{m f}=\beta, b_{m m}=\delta$

From Equation 10 and 11, $b_{f f}=k$ and $b_{m m}=\delta$ can be derived. As a result, the dynamics of the joint, $h_{f f}^{J}$ (TDOF) and $h_{m m}^{J}$ (RDOF), can be obtained as:

$$
\begin{aligned}
H^{J} & =\left[\begin{array}{cc}
h_{f f}^{J} & 0 \\
0 & h_{m m}^{J}
\end{array}\right] \\
& =\left[\begin{array}{cc}
b_{f f}-\left(h_{22, f f}+h_{33, f f}\right) & 0 \\
0 & b_{m m}-\left(h_{22, m m}+h_{33, m m}\right)
\end{array}\right]
\end{aligned}
$$

The joint dynamics are considered to be time invariant parameters, which can be described by the FRF. Then, the curve-fitting algorithm is employed to identify the modal parameters of the joints that can be stored in a database for coupling of arbitrary dynamics in the $\mathrm{FE}$ analysis.

\section{Experimental setup}


To demonstrate the feasibility of the joint identification algorithm, EMA is performed to acquire the FRF by impacting the structure and measuring the vibration responses. In addition, FE analysis was performed to acquire the information on arbitrary Substructure A. Based on the obtained information, an analytic extraction of rotational dynamics at the interface of the modular component is performed and the dynamic characteristics of the joint are retrieved.

Three different test configurations were prepared for impact hammer tests: i) a small cylinder with the machine spindle/tool holder, which represented Substructure B; ii) a rigid cylinder without any joint; and, iii) a cylinder with a fastener joint, as shown in Figure 3(b). The test structures were fabricated with steel (ASTM A108; Young's modulus: 200E9 $\mathrm{N} / \mathrm{m}^{2}$; density: $7800 \mathrm{~kg} / \mathrm{m}^{3}$; damping coefficient: 0.012 ; diameter: $19.95 \mathrm{~mm}$ ). The dimensions of the cylinders, with and without the joint, were identical. The length of the cylinder structure without a joint was $304.8 \mathrm{~mm}$, including the segment $(30 \mathrm{~mm})$ inserted in the collet. As shown in Figure 3, the cylinders, in all cases, were implanted $30 \mathrm{~mm}$ into the collet of the mechanical chuck. The fastener was positioned firmly with sufficient torque to prevent the hysteresis effect [16]; therefore, the effect of the joints will be reflected in the differences of the FRF of the structures.

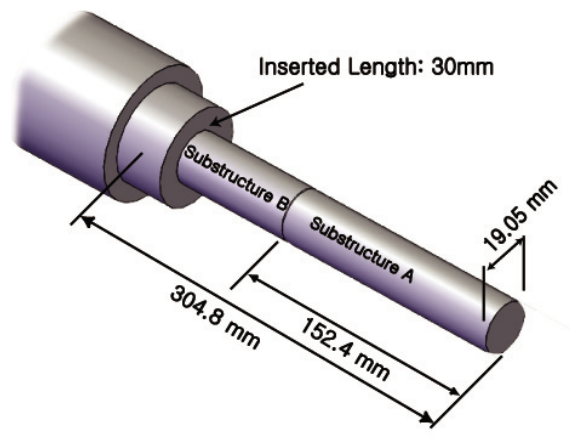

(a)

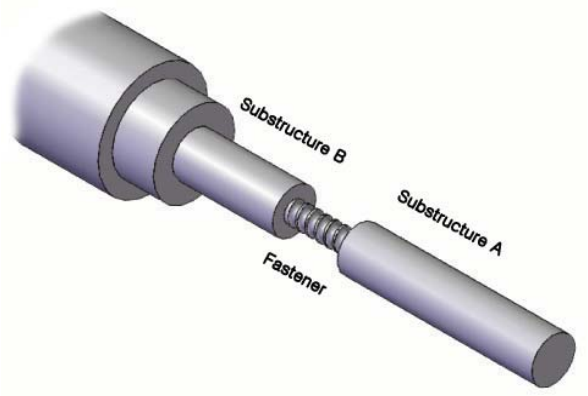

(b)

Figure 3. Dimensions and joint method of the test structures: (a) dimensions of the system, (b) fastener joint

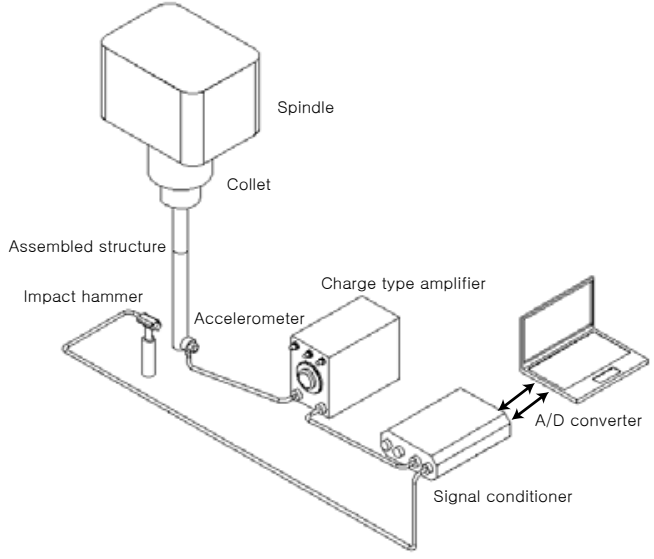

Figure 4. Experimental test setup

In order to define the dynamics of the joints, the FRF are required [9]. Figure 4 illustrates the experimental setup to acquire the experimental FRFs. The structure was excited with the impact hammer (Dytran 58008L), and the response was measured by the accelerometer (Kistler 8778A500) with the mass of $0.29 \mathrm{~g}$. The mass of the accelerometer may affect the measured natural frequencies of the system; therefore, the size of the accelerometer must be chosen carefully. Both signals were fed to a signal conditioner, which had an anti-aliasing filter. The filtered signals were converted from continuous to discrete type by an A/D converter. In this test, only two channels were used to acquire the signals from force and displacement transducers. The converted signals were transformed from the time domain to the frequency domain by the discrete Fourier transform algorithm. From the acquired signals, the transfer functions could be obtained in the frequency domain [9][17]:

$$
H(j \omega)_{p q}=\frac{F(j \omega)_{q}}{X(j \omega)_{p}}
$$

The inherent measurement noise can affect the identified joint dynamics [18]. To minimize the measurement noise (errors), the impact hammer tests were performed at the same location at least seven times and averaged. The measurement data were acquired under a specific sampling rate (i.e. $100 \mathrm{kHz}$ ). Considering the geometry of the structure (short and thick cylinder), the frequency of the FRF should range from 1 to $2,000 \mathrm{~Hz}$, as chosen by the A/D converter. By choosing the high sampling rate and the antialiasing filtering, the aliasing effect could be eliminated. 


\section{Results and discussions}

In this experiment, the effects of the joint for the assembled structure were examined. The joint dynamics were identified by the proposed identification method with data from the experiments and the analytical FE method. Based on the obtained joint dynamics, Substructure B can be mathematically coupled with the new Substructure A, which has arbitrary dimensions and materials. The verification suggests that the mathematically coupled dynamics are in good agreement with the experimental result.

The dynamic responses of each cylinder: one blank cylinder (6 inches in length, Substructure A) and two assembled cylinders ( 6 inches and 5 inches in length, Substructure A) were obtained by the impact hammer test under identical test configurations. Figure 5 shows the dynamic responses of the cylinders at Location $1\left(\mathrm{G}_{11}\right)$.
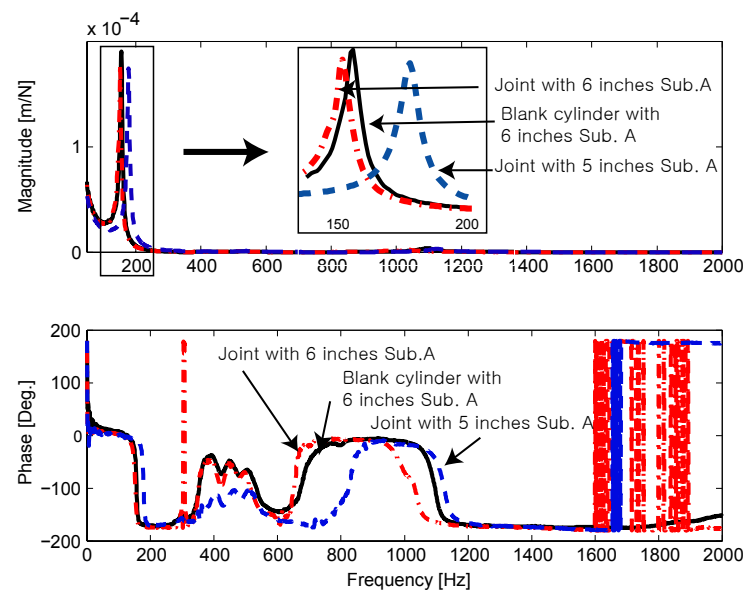

Figure 5. Comparisons between measured frequency responses of each cylinder at $\mathrm{G11}$

The deviations of the dynamic responses of each cylinder were observed. The differences in the dynamic responses between the blank cylinder and the assembled cylinder ( 6 inches, Substructure A) were caused by the dynamic characteristics of the joint. The blank cylinder had a higher natural frequency than the assembled cylinder (6 inches, Substructure A). The result suggests that the presence of the joint decreases the stiffness of the system. Further, deviations in the magnitude at the mode $(1 /(2 k \varsigma))$ of FRF were also observed. The system with a higher damping had the lower magnitude in dynamic response; therefore, the bolted joint had a higher damping, because the interfaces between two substructures were not fully connected. When the bolted joint was forced, slippage at the contact area occurred and generated friction, which results in energy loss.

\subsection{Joint dynamics}

RDOF responses at Location 3 were required prior to the evaluation of the joint dynamics. EMA was performed at Location 3 of Substructure B, and the element, $h_{33, f f}$ (Figure 2), in the transfer function matrix was calculated. In the same manner, in Equation $6,\left[\mathrm{G}_{11}\right]$ and $\left[\mathrm{G}_{12}\right]$ of the rigid cylinder without a joint were measured. It led to RDOF ( $h_{33, m f}$ and $h_{33, m m}$ ) of Location 3 of Substructure B [10]. Similarly, the impact tests were performed on the assembled cylinder (6 inches, Substructure A). Locations 1 and 2 of the assembled structure were stimulated by impact hammer to acquire $\left[\mathrm{G}_{11}\right]$ and $\left[\mathrm{G}_{12}\right]$ (with joint), which were substituted into Equations 10 and 11 with Equation 9. As a result, from Equation 12, the dynamics of the joint, $h_{f f}^{J}$ (TDOF) and $h_{m m}^{J}$ (RDOF), were determined. The identified frequency response of the assembled system by the fastener joint is shown in Figure 6, which is comprised of the real and imaginary parts.

Table 1. The identified joint dynamics expressed in modal mass, modal stiffness, and modal damping.

\begin{tabular}{|c|c|c|c|c|c|}
\hline \multirow[b]{2}{*}{ Modes } & \multicolumn{3}{|c|}{ TDOF: $h_{f f}^{J}$} & \multicolumn{2}{|c|}{ RDOF: $h_{m m}^{J}$} \\
\hline & 1 & 2 & 3 & 1 & 2 \\
\hline $\begin{array}{c}\text { Natural } \\
\text { frequency } \\
\omega_{n}(\mathrm{~Hz})\end{array}$ & 310 & 330 & 440 & 230 & 306 \\
\hline $\begin{array}{c}\text { Modal } \\
\text { stiffness } \\
k(N / m)\end{array}$ & $\begin{array}{c}8.364 \\
\text { E6 }\end{array}$ & $\begin{array}{c}3.026 \\
\text { E6 }\end{array}$ & $\begin{array}{c}16.711 \\
\text { E6 }\end{array}$ & $\begin{array}{c}9.421 \\
\text { E3 }\end{array}$ & $\begin{array}{c}2.747 \\
\text { E7 }\end{array}$ \\
\hline $\begin{array}{l}\text { Modal } \\
\text { Damping } \\
\text { ratio } \zeta\end{array}$ & $\begin{array}{c}0.64 \\
\text { E-2 }\end{array}$ & $\begin{array}{l}5.6 \\
\text { E-2 }\end{array}$ & $\begin{array}{l}0.68 \\
\text { E-2 }\end{array}$ & $\begin{array}{l}1.77 \\
\text { E-2 }\end{array}$ & $\begin{array}{l}1.3 \\
\text { E-2 }\end{array}$ \\
\hline
\end{tabular}



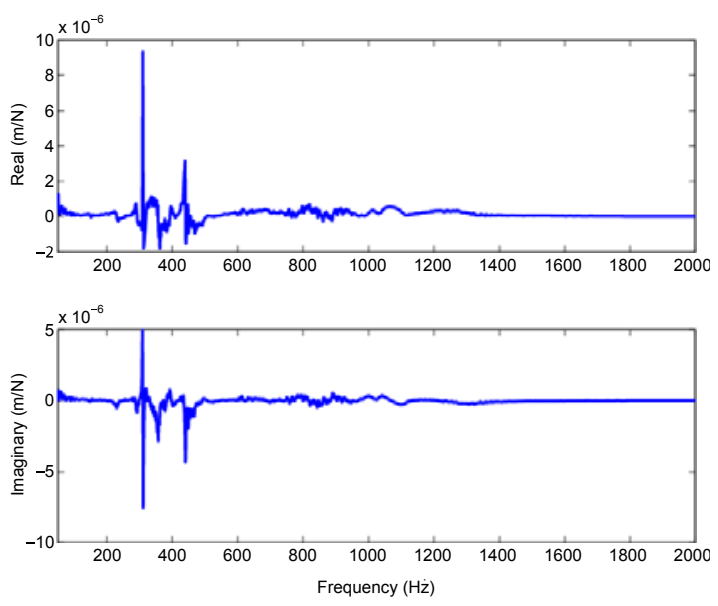

(a)
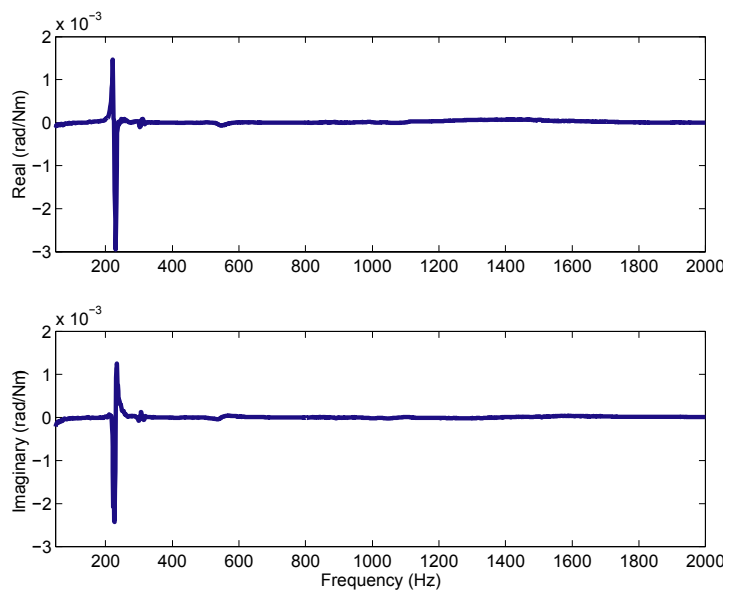

(b)

Figure 6. Identified frequency response of the steel fastener joint: (a) TDOF: $\boldsymbol{h}_{\boldsymbol{f f}}{ }^{J}$, (b) RDOF: $\boldsymbol{h}_{m m}{ }^{J}$

Using modal analysis [9], the dynamic responses shown in Equation 14, such as modal mass, damping ratio and stiffness, can be obtained. Three dominant modes of the joint FRF were selected. The upper and lower boundaries and the algorithm non-linearly were curve fitted using the algorithm [19]. Table 1 depicts the identified modal parameters of the fastener joint dynamics. The parameters are identified based on inhouse developed identification algorithm [21].

$$
\begin{aligned}
h_{f f}^{J}(j \omega) & =\frac{x}{F}=\sum_{i=1}^{3} \frac{1 / m_{i}}{j \omega^{2}+2 \varsigma_{f f, i} \omega_{n, f f, i} j \omega+\omega_{n, f f, i}{ }^{2}} \\
& =\sum_{i=1}^{3} \frac{\omega_{n, i}^{2} / k_{i}}{j \omega^{2}+2 \varsigma_{f f} \omega_{n, i, f f} j \omega+\omega_{n, i, f f}{ }^{2}} \\
h_{m m}^{J}(j \omega) & =\frac{\theta}{M}=\sum_{i=1}^{2} \frac{1 / J_{i}}{j \omega^{2}+2{\varsigma_{m m, i}}_{n, m m, i} j \omega+\omega_{n, m m, i}{ }^{2}} \\
& =\sum_{i=1}^{2} \frac{\omega_{n, i}^{2} / k_{i}}{j \omega^{2}+2 \varsigma_{m m, i} \omega_{n, m m, i} j \omega+\omega_{n, m m, i}{ }^{2}}
\end{aligned}
$$

\subsection{Verifying the identified joint dynamics}

The two assembled cylinders (6 inches and 5 inches in length, Substructure A) consisted of the same joint (fastener) and Substructure B. The dynamic differences in the assembled cylinders also depended on Substructure A, as displayed in Figure 3. In order to verify the identified joint dynamics, the assembled cylinder (5 inches, Substructure A), the FRF was predicted with the identified joint dynamics (Figure 6) and the new receptance matrix for Substructure A from the FE method. Figure 7 depicts the predicted and measured frequency response functions of the assembled cylinder (5 inches, Substructure A).
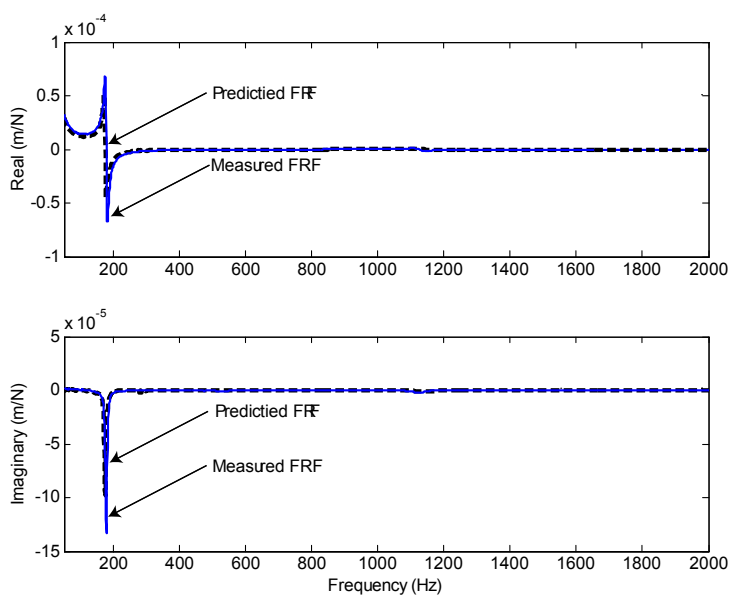

Figure 7. Predicted frequency response of the
assembled cylinder (5 inches, Substructure A)

The deviations of FRF in frequency and magnitude at the first mode between the predicted and measured values varied by approximately $1.68 \%$ and $30 \%$, respectively. This difference was mainly caused by measurement errors, which may have been amplified during the inversion processes. Since the proposed joint identification method is based on experiment data, the existence of measurement noise is inevitable; hence, the filtering algorithm (i.e. SavitzkyGolay Filter) or curve fitting of individual FRF data may be necessary. In addition, the linear assumption for the joint dynamics also contributes to this error, since the friction between the two surfaces may be non-linear and may induce hysteresis phenomenon [7].

\section{Applications}

Considering the particular test environment, this proposed joint identification method can be directly applied to reconfigurable machine tools (RMT). Machine tools should be changed periodically due to 
the wear of the tool, which can depend on the shape and material properties of the workpiece. The screw in a modular tool holder (i.e. a plunge milling cutter [20]) consists of the arbor and the cutting body. The arbor is inserted into the tool holder with the fastener at the end The cutting body has cutters that screw into the fastener of the arbor, depending on the workpiece and the wear of the cutter. For archiving the accuracy of machine performance, the instability (i.e. chatter) of the machine tool must be avoided. To do so, the dynamic characteristics of a machine tool system must be evaluated. It is not effective to perform the dynamic test for the machine tool system whenever the cutter is changed. Once the dynamic characteristics of the joint between the arbor and the cutting body are obtained by the proposed algorithm, engineers can analyze the dynamic characteristics of the machine tool system with the FRF of the cutting body with the FE technique. The evaluation time for the proposed joint identification method in machining tool system is reduced dramatically. This advantage is will be reflected by high productivity of the machining and by cost and time savings.

Further, the dynamic characteristics of various joints can be documented as a database for the conceptual design process. The dynamic properties (i.e. stiffness and damping) of the RMT are critical in achieving manufacturing requirements and various configurations. By assessing the dynamic properties of various joints, designers can accurately evaluate the dynamic properties of the possible configurations of RMT and can select the optimal configurations in the conceptual design stage. Therefore, the proposed joint identification algorithm can accelerate and achieve a low-cost design process.

\section{Summary}

The dynamic characteristics of the joint are essential for accurate evaluations of RMS. This paper presents a novel identification method, which identifies the dynamic characteristics of the joint. The joints were modeled as linear mass, spring and damping elements in the system. Relative equations were derived by improved receptance coupling techniques in combination with the FE method and experimental tests in RDOF and TDOF. The dynamic characteristics of a fastener joint in a mechanical system were identified and verified by the proposed method.

Further verification of the proposed joint identification method can be performed with evaluation of various joints in future research. Take for example, bearings, pin/hole and T-slots in RMS; it is almost impractical to evaluate the dynamics of these joints by the FE method. Therefore, once the dynamic characteristics of the joints are obtained by means of the proposed joint identification method, this method can be applied to variety of applications to predict the overall dynamics of RMS.

This innovative approach overcomes the challenges that other identification methods have encountered and empowers RMT development with the capability of predicting overall modular machine dynamics in the conceptual design stage. This progression in joint identification advances RMT performance towards a cost-effective industry process offers higher accuracy in machining and productivity and, consequently, fulfills the expeditious demand in manufacturing requirements.

\section{References}

[1] Landers, R. G., Min, B.K., and Koren, Y., "Reconfigurable Machine Tools", Annals of the CIRP, 2001, Vol. 50.

[2] Koren, Y., Heisel, U., Jovane, F., Moriwaki, T., Pritschow, G., Ulsoy, G., and Van Brussel, H., "Reconfigurable Manufacturing Systems", Annals of the CIRP, 1999, Vol. 48.

[3] Mehrabi, M. G., Ulsoy, A. G., and Koren, Y., "Reconfigurable manufacturing systems: Key to future manufacturing", Journal of Intelligent Manufacturing, 2000, 11, $403-419$.

[4] Li, H., Landers, R., Kota, S., "A review of feasible joining methods for reconfigurable machine tool components", Japan-USA symposium on flexible automation, Ann Arbor, Michigan, July 23-26, 2000.

[5] Kalpakjian, S., Schmid, S. R., Manufacturing processes for Engineering material, fourth edition, Prentice hall.

[6] Wang, J., Sas, P., "A method for identifying parameters of mechanical joints". Journal of Applied Mechanics, 1990, 57, pp. 337-342.

[7] Shen, J., Astaneh-Asl, A., "Hysteresis model of boltedangle connections". Journal of Constructional steel Research, 2000, 54, pp. 317-343.

[8] Ma, X., Bergman, L., Vakakis, A., "Identification of bolted joints through laser vibrometry", Journal of Sound and Vibration, 2001, 246, pp. 295-316.

[9] Altintas, Y., Manufacturing Automation: Metal cutting mechanics, machine tool vibrations, and CNC design, Cambridge University Press, New York, 2000.

[10] Park, S., Altintas, Y., Movahhedy, M., "Receptance coupling for end mills". Journal of Machine Tools and Manufacture, 2003, 43, pp. 889-896.

[11] Mottershead, J. E., Friswell, M.I., Ng, G.H., and Brandon, J.A., "Geometric parameters for finite element model updating of joints and constraints", Mechanical systems and signal processing, 1996, 10(2), pp. 171-182.

[12] Schmitz, T.L., Davies, M., Kennedy, M.D., "Tool Point Frequency Response Prediction for High-Speed 
Machining by RCSA", Journal of Mfg. Science and Eng., 2002, 123, pp. 700-707.

[13] Song, T., Hartwigsen, C.J., Mcfarland, D.M., Vakakis, A.F., Bergman, L.A., "Simulation of dynamics of beam structures with bolted joints using adjusted Iwan beam elements", Journal of Sound and Vibration, 2004, 273, pp. 249-276.

[14] Hanss, M., Oexl, S., Gaul, L., "Identification of a bolted-joint model with fuzzy parameters loaded normal to the contact interface", Mechanics Research Communications, 2002, 29, pp. 177-187.

[15] Matlab ${ }^{\mathrm{TM}}$, Symbolic Toolbox, The Mathworks, 2001.

[16] Kim, S.M., Ha, J.H., Jeong, S.H., Lee, S.K., "Effect of joint conditions on the dynamic behavior of a grinding wheel spindle". Machine Tools \& Manufacture, 2001, 41, pp. 1749-1761.
[17] Harris, Cyril M., "Material damping and slip damping", Shock and Vibration Handbook, McGraw-Hill, 2, New York, 1997.

[18] Wang, J.H., Chuang, S.C., "Reducing errors in the identification of structural joint parameters using error functions", Journal of Sound and Vibration, 2004, 273, pp. 295-316.

[19] Compomanes, M. L., "Dynamics of Milling Flexible Structures", Ma.Sc. Thesis, University of British Columbia, 1993.

[20] SECO http://www.carboloy.com/products/milling/navpdf/plun geMilling.pdf.

[21] Compomanes, M. L., 1993, "Dynamics of Milling Flexible Structures", Ma.Sc. Thesis, University of British Columbia. 